\title{
La introducción institucional de la e-justicia y la e-administración en Colombia ${ }^{1}$
}

\author{
Alba Luz Serrano Rubiano² \\ Elliot Parra Ávila ${ }^{3}$ \\ Fecha de recepción: 15/06/2020 - Fecha de aceptación: 23/07/2020 \\ DOI : https://doi.org/10.22490/26655489.3927
}

\section{Resumen}

La introducción de la e-administración y la e-justicia en Colombia tienen antecedentes más allá de la actual coyuntura generada por la COVID-19. Para hacer una reflexión ordenada sobre la irrupción de las TIC en la administración y justicia colombianas es necesario exponer la forma en que la e-administración y la e-justicia fueron introducidas institucionalmente al país. Adoptando definiciones académicas de los dos términos analizados, se buscaron normas de carácter general (administrativo y judicial) e instrumentos de planeación nacional o sectorial. La exposición muestra que el desarrollo de la e-administración y la e-justicia en Colombia está en un nivel bajo respecto de la subregión suramericana pero aceptable respecto del mundo. Así mismo, el proceso de la e-administración tiene un recorrido y resultados más amplios que los de la e-justicia. Finalmente, se puede concluir que la cantidad y calidad de los instrumentos de planeación es mayor que la materialización normativa y de gestión que han tenido en la e-administración y la e-justicia.

\footnotetext{
1 Este artículo es producto del proyecto "La formación de juristas en la era digital", ejecutado dentro del grupo de investigación PODET "Política, Derecho y Territorio" (COL0193967), línea de investigación de escuela "Derechos humanos y solución alternativa de conflictos", sublínea "Innovación en la formación jurídico-política" y financiado por la Universidad Nacional Abierta y a Distancia - UNAD.

2 Abogada de la Universidad Cooperativa de Colombia, Magíster en Derecho Administrativo de la Universidad Sergio Arboleda y Decana de la Escuela de Ciencias Jurídicas y Políticas de la Universidad Nacional Abierta y a Distancia - UNAD. ORCID: https://orcid.org/0000-0003-4659-7140 - alba.serrano@unad.edu.co

3 Abogado y Magíster en Derecho de la Universidad Nacional de Colombia, Especialista en Derecho Procesal de la Universidad del Rosario y Master of Research del European University Institute. Docente Asociado de la Universidad Militar Nueva Granada y profesor universitario de pregrado y posgrado, par académico de Conaces y Conjuez del Tribunal Administrativo de Cundinamarca. ORCID: https://orcid.org/0000-0001-63767012. eliot.parra@unad.edu.co
} 
Palabras clave: e-administración colombiana, e-gobierno colombiano, e-justicia colombiana

\section{Abstract}

The introduction of e-administration and e-justice in Colombia has antecedents beyond the current conjuncture generated by COVID-19. To make an orderly reflection on the emergence of ICTs in the Colombian administration and justice, it is necessary to expose the way in which e-administration and e-justice were institutionally introduced to the country. Adopting academic definitions of the two terms analyzed, we search general norms (administrative and judicial) and instruments for national or sectoral planning. The description shows that the development of e-administration and e-justice in Colombia is at a low level with respect to the South American subregion but acceptable with respect to the world. Likewise, the e-administration process has a broader path and results than e-justice. Finally, it can be concluded that the quantity and quality of the planning instruments is greater than the normative and management materialization that they have had in e-administration and e-justice.

Keywords: Colombian e-administration, Colombian e-government, Colombian e-justice

\section{I ntroducción}

La COVID-19 obligó a casi todos los países del mundo a adoptar diferentes niveles de confinamiento o cuarentenas. Esa situación plantea un reto para el normal desarrollo de las funciones del Estado. En Colombia, las más recientes adaptaciones a esta situación han sido los decretos legislativos 491 sobre administración (2020a) y 806 sobre justicia (2020b), que reglamentan la virtualización u ofrecimiento remoto de servicios y procedimientos. Con todo lo que puedan tener de novedosas, estas normas no son la primera expresión de la tecnologización de la administración pública y la administración de justicia colombianas. Para iniciar una reflexión ordenada de la introducción de la e-justicia y la e-administración, este trabajo pretende 
exponer cómo los conceptos de e-justicia y e-administración han sido introducidos institucionalmente en Colombia, al responder la pregunta ¿cómo la institucionalidad colombiana introdujo la e-justicia y la e-administración?

\section{Metodología y procedimientos básicos utilizados}

El enfoque metodológico del trabajo es cualitativo y la herramienta que se empleará será la revisión de literatura de origen oficial (normas y políticas) en busca de conceptos asociados a los objetos estudiados: e-administración y e-justicia.

Para realizar dicha búsqueda se debe hacer una aclaración metodológica: el término amplio del que vienen tanto la e-administración como la e-justicia es el estadounidense e-government. En Estados Unidos el término "gobierno" se usa para designar a los poderes públicos en general. Por esa razón, se suelen confundir las definiciones de e-government con e-administración y la e-justicia se considera una expresión concreta del e-government en general.

Para efecto de este trabajo, empleamos la siguiente definición de e-administración (entendida como la expresión únicamente ejecutiva del "e-government"): "El uso por parte del Gobierno de tecnologías de comunicación de la información para ofrecer a los ciudadanos y las empresas la oportunidad de interactuar y hacer negocios con el Gobierno mediante el uso de diferentes medios electrónicos como el teclado táctil del teléfono, fax, tarjetas inteligentes, quioscos de autoservicio, correo electrónico / I nternet e I ntercambio Electrónico de Datos". (Almarabeh \& AbuAli, 2010, p. 30).

Por su parte, se adoptó la definición de e-justicia como "el gobierno electrónico especializado en temas jurídicos o la inclusión del uso de las tecnologías del conocimiento e información en la administración de justicia. La e-justicia supone el uso de una pluralidad de instrumentos y canales tecnológicos a la hora de impartir justicia" (Bueno de Mata, 2010, p. 3). 
La búsqueda de información se limitó a normas de carácter general (decretos nacionales y leyes de la república) y documentos de planeación (CONPES o Planes de la Rama J udicial) cuyo contenido pudiese satisfacer las definiciones previamente asumidas.

\section{La situación de Colombia en materia de e-Government}

En el año de 2003 las Naciones Unidas, a través de su División de Administración Pública y Gerencia del Desarrollo elaboró el primer estudio mundial sobre gobierno en línea: el e-Government Survey. El informe estaba estructurado alrededor de varios índices que daban cuenta de diversos aspectos del gobierno electrónico en 191 países. El índice general era el de "preparación" del país para el e-gobierno, y estaba compuesto a su vez por tres índices: medición web, infraestructura de telecomunicaciones y capital humano. Para el índice de medición web, Colombia ocupó el puesto 37, para el índice de infraestructura de telecomunicaciones el puesto 79 y para el índice de capital humano ocupó el puesto 100. El resultado final del estudio fue que Colombia ocupaba el puesto 57 entre 191 países en su "preparación" para el e-gobierno (UN Department of Economic and Social Affairs (UNDESA) \& Civic Resource Group (CRG), 2003, p. 61).

Adicionalmente, el informe aportaba otro índice: el de "participación", compuesto de mediciones sobre la capacidad del gobierno para proporcionar a los ciudadanos información, absolver consultas y recibir aportes. En ese índice Colombia ocupó la posición 28 (2003, p. 101). Como nota destacada, el informe alababa que el $94 \%$ de los órganos de la administración nacional (190 de 203) tuviera página web, así como destacó la existencia de un portal unificado de información sobre contratación pública (2003, p. 6)

Trece años después, la metodología del informe varió: el índice de preparación dio paso al índice de desarrollo, mientras que persiste el de participación. Los resultados de Colombia en el informe de 2016 son los siguientes: de 193 países ocupamos el puesto 57 en desarrollo y el puesto 27 en participación (UN Department of Economic and Social Affairs (UNDESA), 2016). La situación colombiana se puede apreciar en la siguiente ilustración: 


\section{I lustración 1. Í ndice e-government para Colombia}

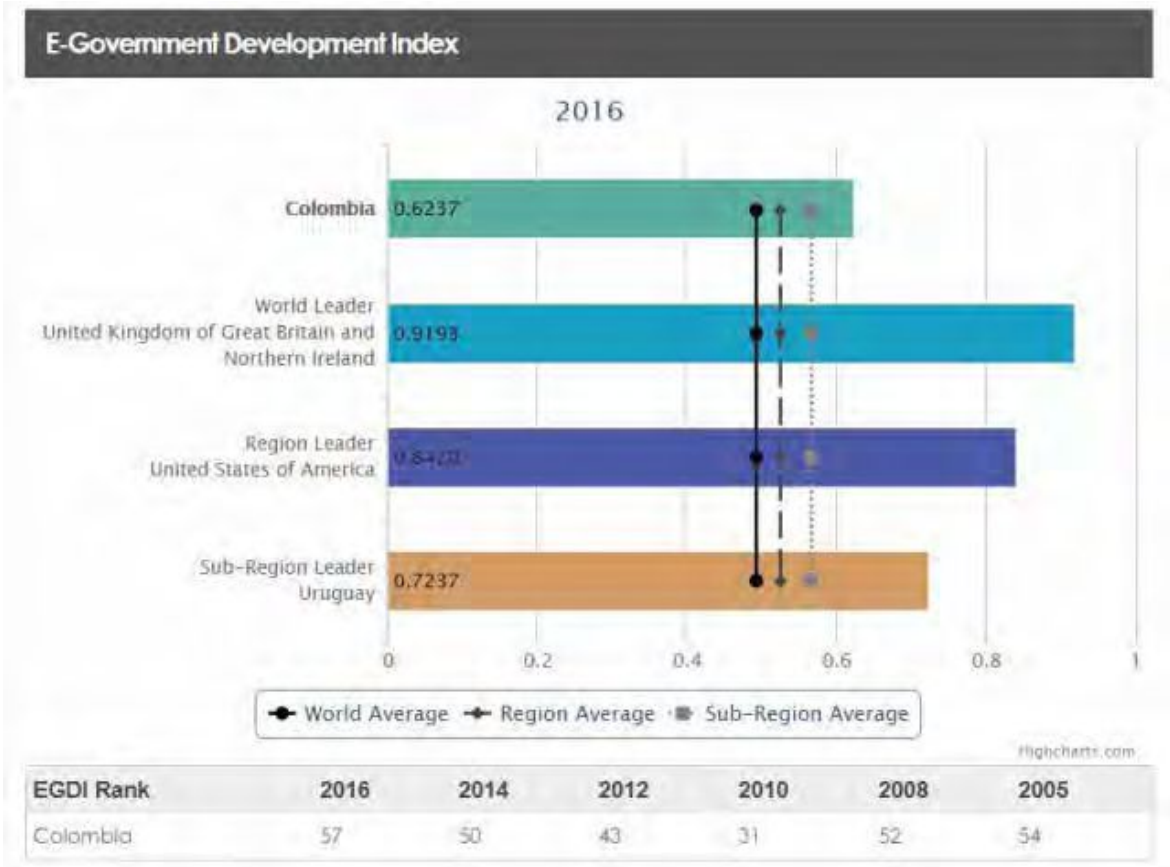

Fuente: (UNDESA, 2016)

Como se aprecia, Colombia alcanzó su mejor desempeño en 2010, pero a la privilegiada posición 31 le siguió un deterioro que ha enviado al país a niveles similares a los de 2003 (o peores, si se considera que en 2016 participaron en la medición 193 países). Aunque el país está por delante de los promedios regional y subregional, en perspectiva histórica los resultados están lejos de ser buenos.

En lo que respecta a la participación, el desempeño del país es mejor en términos generales, con el liderazgo a nivel subregional, pero también muestra una pérdida del terreno ganado entre 2012 y 2014 y un retroceso a los niveles de 2003, tal como se puede apreciar en la siguiente ilustración: 


\section{I lustración 2. Í ndice e-participation para Colombia 2016 (UNDESA)}

\section{E-Parlicipalion Index}

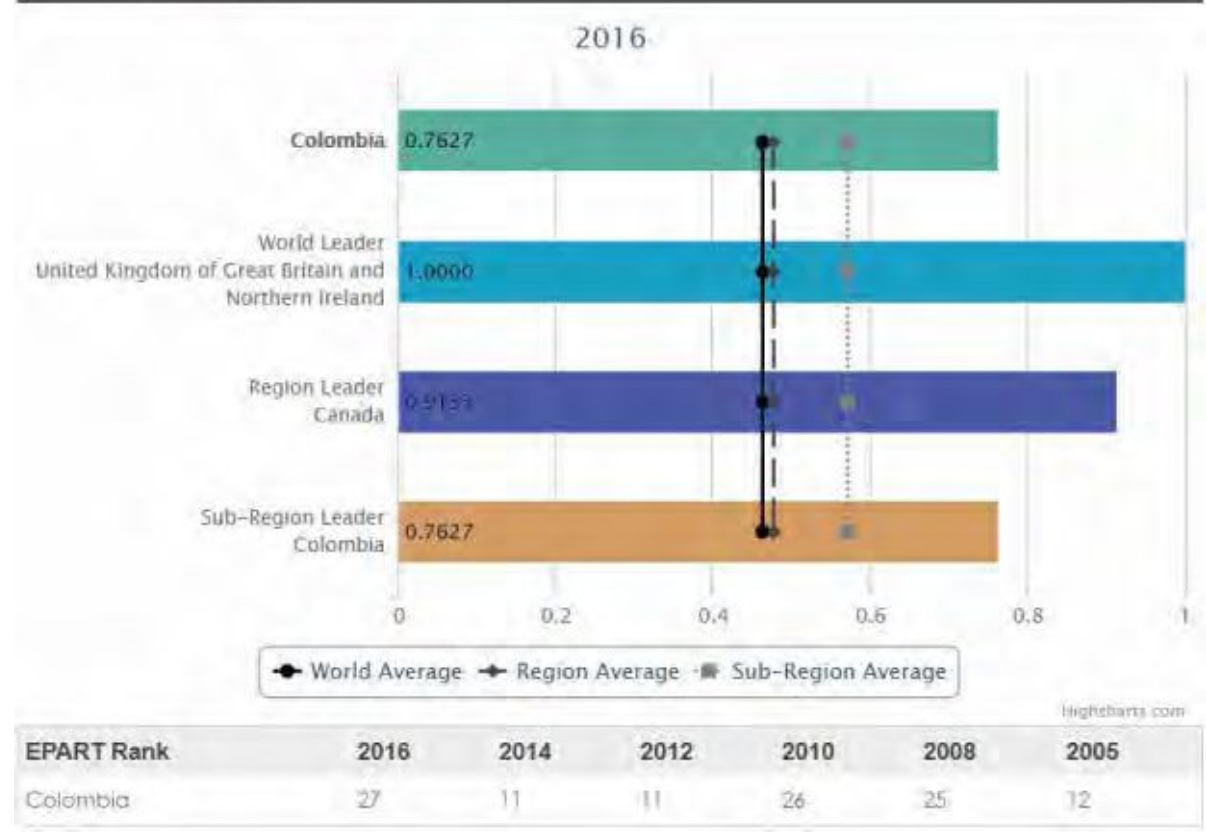

Fuente: (UNDESA, 2016)

De especial importancia es el desglose de los componentes del índice de desarrollo del e-government, que en la metodología actual son tres: índice de servicios en línea (reemplazó a la medición web), infraestructura de telecomunicaciones y capital humano, como se aprecia a continuación: 


\section{I lustración 3. Í ndice de servicio en línea para Colombia 2016 (UNDESA)}

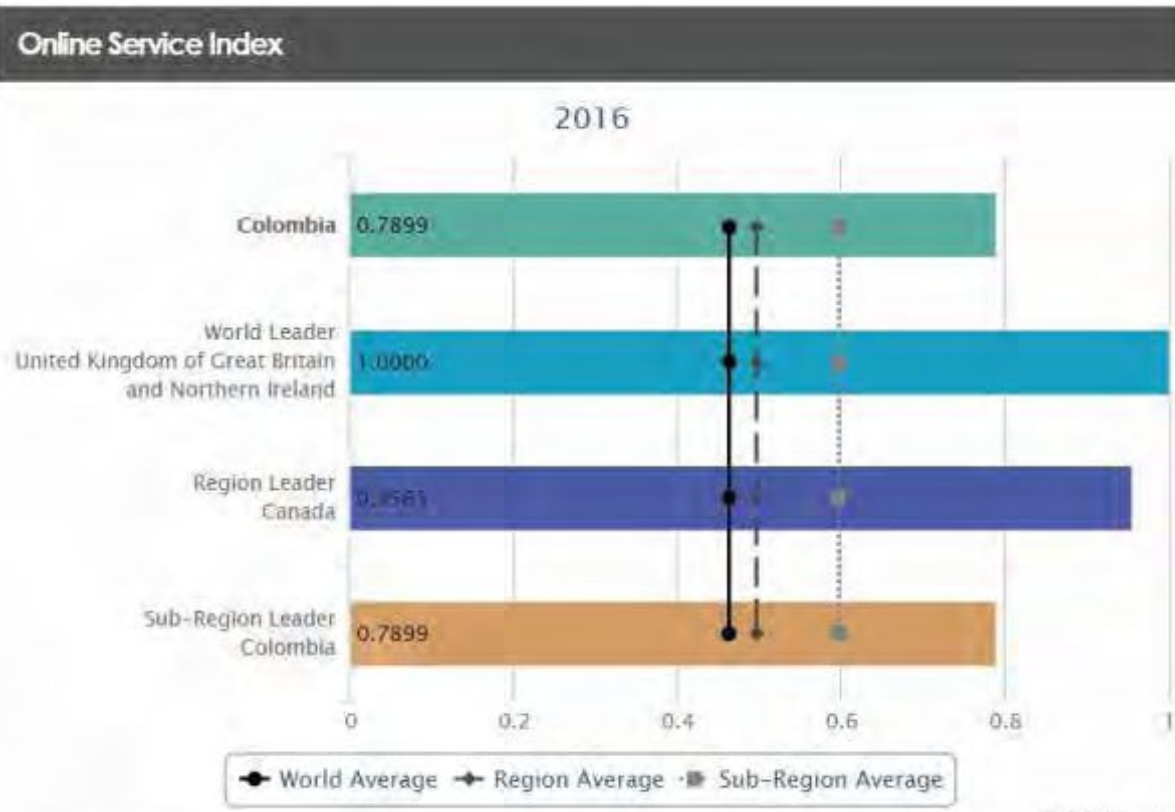

Fuente: (UNDESA, 2016)

Para el informe, la definición de este índice es "un indicador compuesto que mide el uso de las TIC por parte de los gobiernos para prestar servicios públicos a nivel nacional" (2016, p. 79). En este índice Colombia es la líder subregional, con un desempeño superior en un $30 \%$ al promedio regional y en un $25 \%$ al promedio subregional, lo que indica que la administración pública del país hace gran uso de las TIC en su gestión.

Respecto a la infraestructura de telecomunicaciones el resultado es el siguiente: 


\section{I lustración 4. Í ndice de infraestructura de telecomunicaciones para Colombia.}

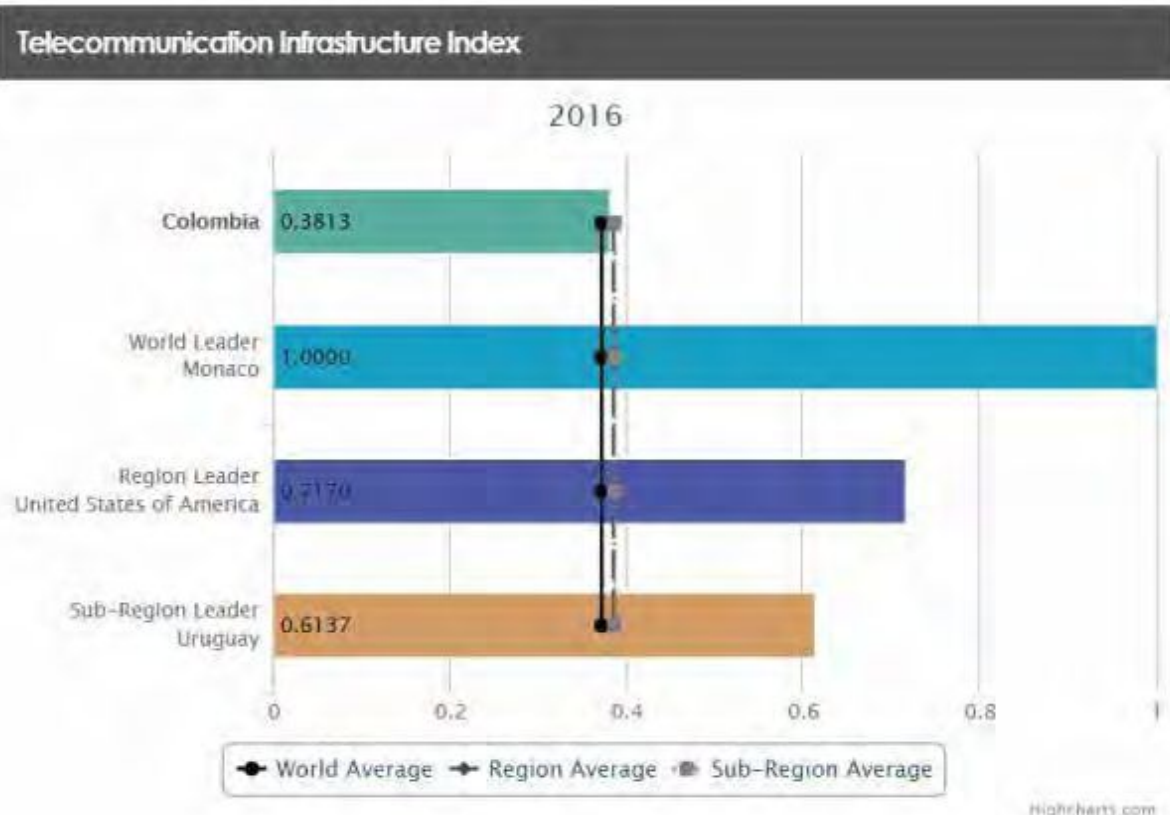

Fuente: (UNESA, 2016)

Como era de esperarse, el desempeño es insatisfactorio y el país se ve casi que duplicado por el líder subregional, y se encuentra sobre los promedios mundial y regional. Ésto indica que aún queda mucho por avanzar en el tema.

Para el índice de capital humano, que involucra la medición de:

i. Tasa de alfabetización de adultos.

ii. La tasa bruta combinada de matrícula primaria, secundaria y terciaria.

iii. Años esperados de enseñanza.

iv. Años promedio de escolaridad (2016, p. 136).

Debemos recordar que proviene de un índice externo: el Índice de Desarrollo Humano (IDH) que también lleva a cabo la ONU y sus resultados se ilustran a continuación: 


\section{I lustración 5. Í ndice de capital humano para Colombia.}

\section{Human Capilial Index}

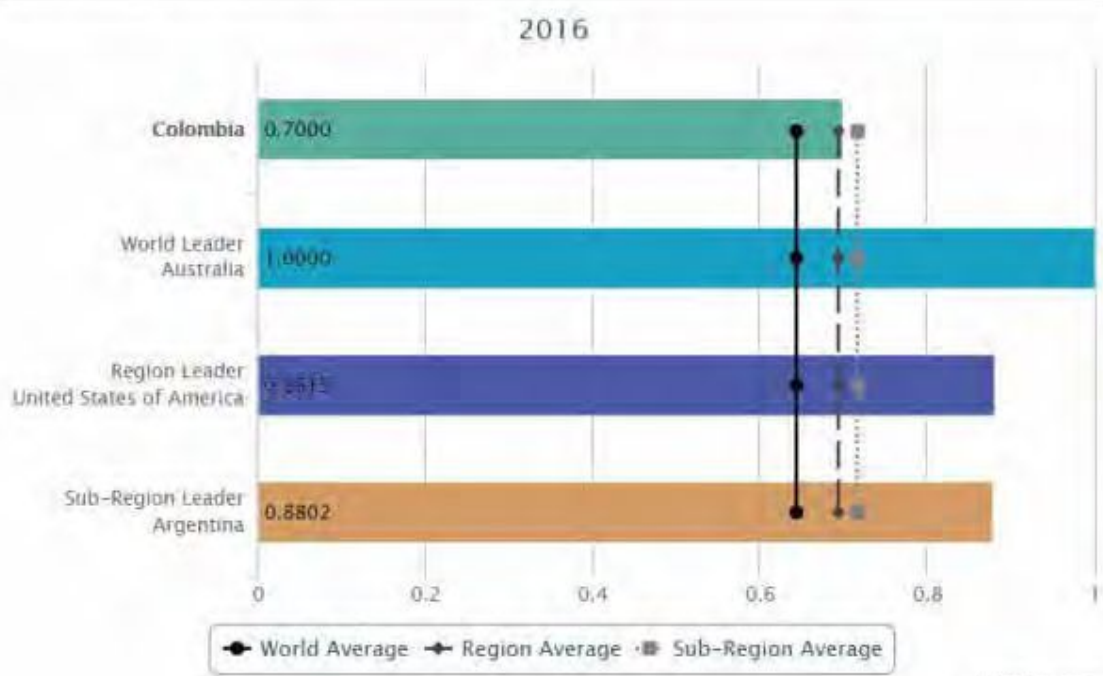

Fuente: (UNDESA, 2016)

Aunque Colombia se encuentra por encima del promedio mundial, apenas está en el promedio regional y se encuentra por debajo tanto del promedio como del líder subregional, lo que evidencia que el país aún está en mora de mejorar la calidad de su educación, de acuerdo con la composición eminentemente educativa de este índice.

\section{Los elementos institucionales colombianos de la e-administración (2000-2015)}

¿Cuál ha sido la respuesta del gobierno colombiano al déficit de gobernanza digital descrito en el contexto global? Para seguir la línea de tiempo planteada, en el 2000 fue publicado el Documento Conpes 3072 "Agenda de conectividad", en el que se identificaban tres sectores para ser impactados por ella: la comunidad, el sector productivo y el Estado. Respecto a este último sector (el estatal), el documento Conpes 3072 describía el ambiente favorable para la agenda como un cambio cultural en los órganos estatales que buscaba: 
“garantizar la adopción de las tecnologías de la información al interior de estos. Como complemento a lo anterior, se revisarán y optimizarán los procesos internos de las entidades y se establecerán esquemas estandarizados para intercambio de información a nivel interinstitucional. De otra parte, el Gobierno determinará un marco legal que garantice la regulación de todos los aspectos relacionados con el uso de las tecnologías de la información, y dispondrá de mecanismos orientados al acercamiento con la comunidad" (Consejo Nacional de Política Económica y Social, 2000, p. 16)

Para ello se establecieron seis estrategias: acceso a la infraestructura de la información; uso de $\mathrm{TI}$ en los procesos educativos y capacitación en el uso de $\mathrm{TI}$; uso de $\mathrm{TI}$ en las empresas, fomento a la industria nacional de TI; generación de contenido y la última de ellas, Gobierno en Línea, en adelante GEL (2000, p. 17).

A la estrategia GEL se le estructuró inicialmente con los siguientes programas: creación de una intranet gubernamental; creación del portal de Internet con información y servicios gubernamentales; capacitación en el uso de TI para empleados públicos; red de información oficial para la evaluación y seguimiento de la gestión de las entidades del Estado; aplicación de TI en el Gobierno para prestar un mejor servicio al ciudadano; sistema de gestión presidencial y sistema de presupuesto nacional (2000, p. 21).

En ese contexto fue expedida la Ley 1349 (2009) que definió los principios de la sociedad de la información y reguló las TIC. En el ámbito jurídico, la ley estableció que:

“La investigación, el fomento, la promoción y el desarrollo de las Tecnologías de la Información y las Comunicaciones son una política de Estado que involucra a todos los sectores y niveles de la administración pública y de la sociedad, para contribuir al desarrollo educativo, cultural, económico, social y político e incrementar la productividad, la competitividad, el respeto a los derechos Humanos inherentes y la inclusión social" (artículo 2).

Ya consagrado el respeto por los derechos humanos como un derrotero, contempló la protección de los derechos 
de los usuarios ${ }^{4}$ y la masificación del $\mathrm{GEL}^{5}$ como principios orientadores. Así mismo, impuso deberes a las entidades nacionales y territoriales para intensificar los servicios ofrecidos a través de las TIC $^{6}$ y consagró protección jurídica de los derechos involucrados cuando se emplean dichas tecnologías: el de rectificación ${ }^{7}$ y el de confidencialidad ${ }^{8}$.

La estrategia GEL se mantuvo en el tiempo y en la actualidad está regulada por el Decreto 1078 (2015). Lo primero que ha de anotarse es que GEL está sometida a los mismos principios de toda función administrativa: debido proceso, igualdad, imparcialidad, buena fe, moralidad, participación, responsabilidad, transparencia, publicidad, coordinación, eficacia, economía y celeridad (artículo 2.2.9.1.1.4.). Los cuatro componentes de GEL son los siguientes.

Componentes. Los fundamentos de la Estrategia serán desarrollados a través de 4 componentes que facilitarán la masificación de la oferta y la demanda del Gobierno en Línea.

a. TIC para servicios. Comprende la provisión de trámites y servicios a través de medios electrónicos, enfocados en dar solución a las principales necesidades y demandas de los ciudadanos y empresas, en condiciones de calidad, facilidad de uso y mejoramiento continuo.

4 "El Estado velará por la adecuada protección de los derechos de los usuarios de las Tecnologías de la Información y de las Comunicaciones, así como por el cumplimiento de los derechos y deberes derivados del Hábeas Data, asociados a la prestación del servicio [...]" (artículo 2, numeral 4).

5 "Con el fin de lograr la prestación de servicios eficientes a los ciudadanos, las entidades públicas deberán adoptar todas las medidas necesarias para garantizar el máximo aprovechamiento de las Tecnologías de la Información y las Comunicaciones en el desarrollo de sus funciones. El Gobierno nacional fijará los mecanismos y condiciones para garantizar el desarrollo de este principio [...]" (artículo 2, numeral 8).

6 “[...] Parágrafo $1 .{ }^{\circ}$. Las entidades de orden nacional y territorial incrementarán los servicios prestados a los ciudadanos a través del uso de las Tecnologías de la Información y las Comunicaciones. El Gobierno reglamentará las condiciones en que se garantizará el acceso a la información en línea, de manera abierta, ininterrumpida y actualizada, para adelantar trámites frente a entidades públicas, inclusive en el desarrollo de procesos de contratación y el ejercicio del derecho al voto" (artículo 5).

7 "Derecho de rectificación. El Estado garantizará el derecho de rectificación a toda persona o grupo de personas que se considere afectado por informaciones inexactas que se transmitan a través de los servicios de telecomunicaciones, sin perjuicio de las acciones civiles, penales y administrativas a que hubiere lugar. (artículo 70).

8 "Confidencialidad. El Estado garantizará la inviolabilidad, la intimidad y la confidencialidad en las Telecomunicaciones, de acuerdo con la Constitución y las leyes." (artículo 71). 
b. TIC para el Gobierno abierto. Comprende las actividades encaminadas a fomentar la construcción de un Estado más transparente, participativo y colaborativo involucrando a los diferentes actores en los asuntos públicos mediante el uso de las Tecnologías de la Información y las Comunicaciones.

c. TIC para la Gestión. Comprende la planeación y gestión tecnológica, la mejoría de procesos internos y el intercambio de información. Igualmente, la gestión y aprovechamiento de la información para el análisis, toma de decisiones y el mejoramiento permanente, con un enfoque integral para una respuesta articulada de gobierno y para hacer más eficaz la gestión administrativa entre instituciones de Gobierno.

d. Seguridad y privacidad de la Información. Comprende las acciones transversales él los demás componentes enunciados, tendientes a proteger la información y los sistemas de información, del acceso, uso, divulgación, interrupción o destrucción no autorizada (artículo 2.2.9.1.2.1).

Los proyectos a través de los cuales se ejecutan los componentes del GEL son: la Ruta de la Excelencia, el Sello de la Excelencia, Servicios Digitales Básicos, Programa para la Excelencia del Gobierno Digital, "No más filas", "Mi Colombia digital", Urna de Cristal y Datos Abiertos (Ministerio de Tecnologías de la Información y las Comunicaciones, 2017).

En este estado de cosas fue expedida la Ley Estatutaria 1581 (2012b) sobre protección de datos personales, que reguló integralmente la materia, asignando responsabilidades sobre los datos (como se verá más abajo), incorporando nuevos principios sobre protección de datos: principio de legalidad en materia de tratamiento de datos, principio de finalidad, principio de libertad, principio de veracidad o calidad, principio de transparencia, principio de acceso y circulación restringida, principio de seguridad y principio de confidencialidad (artículo 4); estableciendo nuevas categorías de datos como los sensibles y los de menores y adolescentes (artículos 5 al 7), regulando procedimientos de consulta y reclamación (artículos 14 al 16), estableciendo deberes a los responsables y encargados del tratamiento de datos (artículos 17 y 18) y todo un procedimiento sancionatorio (artículos 22 al 24). 


\section{La introducción de la e-justicia (2002-2012)}

La historia de la adopción de las TIC por parte de la rama judicial es más reciente que la gubernamental. En el Plan de Desarrollo Sectorial de la rama judicial 2003-2006 (Consejo Superior de la Judicatura, 2002) se proponían dos políticas relacionadas con las TIC, aunque sin asumir esa denominación

"Continuar los procesos de modernización de la infraestructura física, de los sistemas de archivo, y de la infraestructura informática y telemática de la administración de justicia.

Impulsar nuevos instrumentos procesales que agilicen la gestión judicial con mecanismos como la oralidad y el aprovechamiento de herramientas informáticas y telemáticas" (2002, p. 24).

La ejecución de esas políticas se proponía bajo el programa ( $\sin$ estrategia) denominado "Modernización y desarrollo de la infraestructura tecnológica" que se realizaba a través de dos proyectos, el "2.4.1.1 Sistematización de los despachos judiciales y las dependencias administrativas" y el "2.4.1.2 Desarrollo, integración e implementación de servicios telemáticos para la conformación de la Red informática judicial; fortaleciendo tanto la comunicación interna como con la ciudadanía" (2002, p. 30).

Para el momento en que se emitió el Plan Sectorial 20072010 (Consejo Superior de la Judicatura, 2006) la evaluación que se hacía del anterior, sin aportar cifras, era de logros como la "ampliación de nuevos servicios virtuales a través del portal web de la rama judicial: consulta de los auxiliares de la justicia, sistema de fondos especiales y cobro coactivo, sistema de administración de carrera judicial, sistema de información de personal de la rama judicial y sistema de auditoría de la rama judicial" (2006, p. 43).

No obstante esa afirmación, el propio plan reconocía la existencia de una "débil coordinación interinstitucional en gestión, en administración de información y en comunicaciones" (p. 56) y para solucionarla proponía, dentro de las políticas de eficiencia y eficacia "ampliar la cobertura y fortalecer los procesos de modernización de la infraestructura física, de los sistemas de archivo y de la infraestructura informática y telemática de la administración de justicia" (p. 68) y dentro de las políticas de comunicaciones "promover el desarrollo gradual de sistemas 
virtuales y de comunicaciones electrónicas en la administración de justicia en lo jurisdiccional y administrativo, orientados a la optimización de trámites" (p. 73). Como se ve, las TIC eran percibidas más como un problema de archivística interna que como una forma de gestión judicial.

La evaluación de este plan que se haría cuatro años después en el Plan Sectorial 2011-2014 (Consejo Superior de la Judicatura, 2011) que exponía tímidamente la "incursión en audiencias virtuales entre otros logros" ( $p$. 8) y como un logro en materia de calidad reivindicaba la "capacitación en informática y telemática" de sujetos indeterminados (2011, p. 34). Mientras se reconocía que el problema de la eficiencia en la "gestión judicial" estaba conectado con la oralidad, el "déficit en tecnología y comunicaciones" era un asunto de "comunicación de las decisiones judiciales al ciudadano" (p. 48).

Como política de eficiencia y eficacia se proponía la “modernización de la infraestructura tecnológica acorde con los cambios en el sistema judicial para disponer de una cobertura adecuada en hardware, software, aplicativos especializados y sistemas de comunicación para el sistema judicial escrito y oral mediante la introducción del expediente digital, la notificación electrónica en el trámite procesal [...]" (2011, p. 83). Desde otro enfoque, una de las tres políticas transversales era la:

Política de Tecnologías de la Información y la Comunicación para mejoramiento del acceso a la justicia, la eficiencia en la gestión judicial, la calidad de la respuesta de la justicia, la confianza visibilidad y transparencia y para el fortalecimiento institucional de cara al ciudadano (2011, p. 87).

Hasta aquí se puede observar un diagnóstico repetitivo, unas medidas similares y un aparente bajo impacto de las medidas en el diagnóstico, con una innovación en la perspectiva transversal del plan de 2011. 
Por la época de ejecución del Plan Sectorial 2007-2010, el Ministerio de Comunicaciones señalaba en su Plan Nacional TIC - PNTIC (2008) que se debían emprender acciones como:

“[...] lograr el cubrimiento total del sistema judicial, incluido el nivel regional, de la infraestructura para uso e interconexión a través de las TIC; el funcionamiento de un sistema integrado de información para la gestión judicial; lograr la interoperabilidad a través de las TIC de las entidades directamente relacionadas con el proceso de justicia en los tres poderes públicos, como son entre otras, la Fiscalía General de la Nación, la Policía Nacional, los despachos judiciales, el Inpec, la Defensoría del Pueblo, la Procuraduría General de la Nación, la Registraduría Nacional del Estado Civil, el ICBF, las notarías y las cámaras de comercio; hacer una adecuada gestión de recursos humanos de la rama judicial, en particular de las personas que desarrollan la carrera judicial; y la investigación, la vigilancia tecnológica y divulgación de nuevas metodologías y técnicas para el uso de TIC en la justicia, todo ello en un marco de políticas de seguridad muy estrictas" (2008, p. 73).

En la práctica estaba casi todo por hacer, y mucho más si se asumía una visión integral de la gestión jurídica que involucrara a entidades administrativas que necesariamente, a través del intercambio de información, hacen posible la efectividad de la justicia. Muy importante es la observación que hace el Ministerio a renglón seguido: "como condición para que las acciones mencionadas sean efectivas, se hace necesario sensibilizar y proveer entrenamiento a los funcionarios judiciales en el uso de las TIC". Es decir que el PNTIC anunciaba un perfil específico de labores judiciales que necesariamente combina competencias tecnológicas (TIC) y jurídicas (judiciales).

Los objetivos e indicadores guía del eje justicia se aprecian en la siguiente tabla del Ministerio de Comunicaciones: 


\section{Tabla 2: Objetivos e indicadores guía del eje justicia}

\begin{tabular}{|c|c|c|c|c|c|c|}
\hline Objetivo & $\begin{array}{l}\text { Indicador } \\
\text { principal }\end{array}$ & 2006 & 2007 & $\begin{array}{l}\text { Meta } \\
2010\end{array}$ & $\begin{array}{l}\text { Meta } \\
2019\end{array}$ & Fuente \\
\hline \multirow{6}{*}{$\begin{array}{l}\text { Lograr un ejercicio } \\
\text { eficiente de la } \\
\text { función judicial } \\
\text { y administrativa } \\
\text { de los despachos } \\
\text { judiciales en } \\
\text { todos los niveles } \\
\text { y para todas } \\
\text { las actuaciones, } \\
\text { para aumentar } \\
\text { la productividad } \\
\text { y el rendimiento } \\
\text { de los despachos } \\
\text { judiciales y } \\
\text { mejorar la calidad } \\
\text { del servicio } \\
\text { público, básico } \\
\text { y esencial de } \\
\text { administración } \\
\text { pública. }\end{array}$} & $\begin{array}{l}\text { Funcionamiento } \\
\text { de un único } \\
\text { sistema de } \\
\text { información de } \\
\text { gestión judicial, } \\
\text { que integre toda } \\
\text { la funcionalidad } \\
\text { de la gestión } \\
\text { judicial, en } \\
\text { todos los } \\
\text { juzgados y } \\
\text { tribunales. }\end{array}$ & N.D. & N.D. & $50 \%$ & $100 \%$ & PNTIC \\
\hline & $\begin{array}{l}\text { Integración de } \\
\text { los sistemas } \\
\text { de información } \\
\text { que conforman } \\
\text { o participan } \\
\text { dentro del } \\
\text { proceso de } \\
\text { gestión judicial. }\end{array}$ & N.D. & N.D. & $100 \%$ & $100 \%$ & PNTIC \\
\hline & $\begin{array}{l}\text { Depuración de } \\
\text { la calidad de } \\
\text { la información } \\
\text { del sistema de } \\
\text { información de } \\
\text { gestión judicial } \\
\text { (no duplicidad e } \\
\text { identificación de } \\
\text { los datos reales } \\
\text { de los sujetos } \\
\text { procesales a } \\
\text { través de cruce } \\
\text { de información } \\
\text { con otras } \\
\text { entidades, entre } \\
\text { otros aspectos). }\end{array}$ & N.D. & N.D. & $30 \%$ & $100 \%$ & PNTIC \\
\hline & $\begin{array}{l}\text { Disponibilidad } \\
\text { de herramientas } \\
\text { de planeación y } \\
\text { gestión para los } \\
\text { funcionarios. }\end{array}$ & N.D. & N.D. & $50 \%$ & $100 \%$ & PNTIC \\
\hline & $\begin{array}{l}\text { Disponibilidad } \\
\text { de estadísticas } \\
\text { judiciales } \\
\text { confiables, en } \\
\text { tiempo real. }\end{array}$ & N.D. & N.D. & $100 \%$ & $100 \%$ & PNTIC \\
\hline & $\begin{array}{l}\text { Disponibilidad } \\
\text { de un sistema } \\
\text { de información } \\
\text { gerencial, para } \\
\text { la toma de } \\
\text { decisiones. }\end{array}$ & N.D. & N.D. & $50 \%$ & $100 \%$ & PNTIC \\
\hline
\end{tabular}




\begin{tabular}{|c|c|c|c|c|c|c|}
\hline Objetivo & $\begin{array}{l}\text { Indicador } \\
\text { principal }\end{array}$ & 2006 & 2007 & $\begin{array}{l}\text { Meta } \\
2010\end{array}$ & $\begin{array}{l}\text { Meta } \\
2019 \\
\end{array}$ & Fuente \\
\hline $\begin{array}{l}\text { Dar acceso } \\
\text { inmediato y } \\
\text { en línea de los } \\
\text { usuarios al servicio } \\
\text { de justicia vía } \\
\text { electrónica y } \\
\text { acceso de todos } \\
\text { los ciudadanos a } \\
\text { cualquier tipo de } \\
\text { información judicial } \\
\text { permitido por la } \\
\text { ley (e-justicia). }\end{array}$ & $\begin{array}{l}\text { Sistema de } \\
\text { información } \\
\text { en línea en } \\
\text { funcionamiento. }\end{array}$ & N.D. & N.D. & $50 \%$ & $100 \%$ & PNTIC \\
\hline $\begin{array}{l}\text { Ejercer en forma } \\
\text { oficial y pública } \\
\text { los controles } \\
\text { de gestión, } \\
\text { rendimiento y } \\
\text { resultados de la } \\
\text { actividad de todos } \\
\text { los servidores de } \\
\text { la rama judicial } \\
\text { para construir } \\
\text { el conocimiento } \\
\text { científico sobre las } \\
\text { cargas de trabajo } \\
\text { y la demanda } \\
\text { de justicia, que } \\
\text { permita consolidar } \\
\text { la carrera judicial. }\end{array}$ & $\begin{array}{l}\text { Sistema de } \\
\text { información } \\
\text { en línea en } \\
\text { funcionamiento. }\end{array}$ & N.D. & N.D. & $50 \%$ & $100 \%$ & PNTIC \\
\hline $\begin{array}{l}\text { Permitir la } \\
\text { interacción de los } \\
\text { abogados litigantes } \\
\text { con los despachos } \\
\text { judiciales, a } \\
\text { través de una red } \\
\text { Judicial nacional } \\
\text { para obtener } \\
\text { seguridad jurídica, } \\
\text { confidencialidad, } \\
\text { veracidad y certeza } \\
\text { procesal, ahorro de } \\
\text { tiempo, reducción } \\
\text { de costos, } \\
\text { organización y } \\
\text { transparencia } \\
\text { procesal. }\end{array}$ & $\begin{array}{l}\text { Sistema de } \\
\text { información } \\
\text { en línea en } \\
\text { funcionamiento. }\end{array}$ & N.D. & N.D. & $50 \%$ & $100 \%$ & PNTIC \\
\hline \multirow{2}{*}{$\begin{array}{l}\text { Conservar la } \\
\text { memoria judicial } \\
\text { como patrimonio } \\
\text { histórico de la } \\
\text { nación. }\end{array}$} & $\begin{array}{l}\text { Memoria judicial } \\
\text { digitalizada. }\end{array}$ & N.D. & N.D. & $50 \%$ & $100 \%$ & PNTIC \\
\hline & $\begin{array}{l}\text { Penitenciaria y } \\
\text { cárceles INPEC } \\
\text { conectadas por } \\
\text { el programa } \\
\text { Comparte }\end{array}$ & 2 & 2 & $100 \%$ & $20 \%$ & PNTIC \\
\hline
\end{tabular}




\begin{tabular}{|c|l|c|c|c|c|c|}
\hline Objetivo & $\begin{array}{c}\text { Indicador } \\
\text { principal }\end{array}$ & $\mathbf{2 0 0 6}$ & $\mathbf{2 0 0 7}$ & $\begin{array}{c}\text { Meta } \\
\mathbf{2 0 1 0}\end{array}$ & $\begin{array}{c}\text { Meta } \\
\mathbf{2 0 1 9}\end{array}$ & Fuente \\
\hline & $\begin{array}{l}\text { Porcentaje } \\
\text { de tribunales } \\
\text { superiores } \\
\text { sistematizados. }\end{array}$ & & $90 \%$ & $100 \%$ & $100 \%$ & $\begin{array}{c}\text { CSJ- } \\
\text { PNTIC }\end{array}$ \\
\cline { 1 - 5 } & $\begin{array}{l}\text { Porcentaje } \\
\text { de tribunales } \\
\text { administrativos } \\
\text { sistematizados. }\end{array}$ & & $94 \%$ & $100 \%$ & $100 \%$ & $\begin{array}{c}\text { CSJ- } \\
\text { PNTIC }\end{array}$ \\
\cline { 4 - 7 } & $\begin{array}{l}\text { Porcentaje } \\
\text { de juzgados } \\
\text { capitales } \\
\text { sistematizados }\end{array}$ & $96 \%$ & $100 \%$ & $100 \%$ & $\begin{array}{c}\text { CSJ- } \\
\text { PNTIC }\end{array}$ \\
\hline
\end{tabular}

Fuente: (Mincomunicaciones, 2008)

Excepto por la sistematización de los tribunales, ninguno de los porcentajes señalados para 2010 se ha cumplido a diciembre de 2019. No obstante, la rama judicial respondió al PNTIC y a la exigencia del Código General del Proceso ${ }^{9}$ (2012a) con el Plan Estratégico Tecnológico (Consejo Superior de la J udicatura, 2012) con un marco temporal de seis (6) años.

El Plan Estratégico Tecnológico - PET propone cinco ejes estratégicos para la acción:

a. Modelo de expediente electrónico, con actuaciones en definición y especificaciones técnicas del nuevo expediente electrónico, proyecto de implementación y transición en materia de expediente electrónico y proyecto de modelo jurídico con el fin de asegurar la adecuada tramitación de los procesos escritos y orales.

b. Justicia en Red, con actuaciones en el programa interno (intranet) y programa externo (plan de comunicaciones e Interoperabilidad gestión política y gestión técnica).

c. Gestión de la información, con los proyectos de integración de las bases de datos, para la generación

9 "El Plan de Justicia Digital estará integrado por todos los procesos y herramientas de gestión de la actividad jurisdiccional por medio de las tecnologías de la información y las comunicaciones, que permitan formar y gestionar expedientes digitales y el litigio en línea. El plan dispondrá del uso obligatorio de dichas tecnologías de manera gradual, por despachos judiciales o zonas geográficas del país, de acuerdo con la disponibilidad de condiciones técnicas para ello" (Ley 1564, artículo 103). 
y tratamiento de las estadísticas judiciales, de medios tecnológicos para apoyar la rendición de cuentas, sobre aspectos normativos vinculados a la gestión de la información, para la generación de conocimiento a partir de las bases de datos de normatividad, doctrina y jurisprudencia, para la incorporación de herramientas y para la mejora de la accesibilidad de las personas con discapacidad.

d. Gestión del cambio, con la elaboración de una estrategia integral de gestión del cambio y los proyectos de capacitación y sensibilización de los diversos actores y de la ciudadanía, de adecuación de infraestructuras, de adecuación de los modelos de gestión, de fortalecimiento y actualización del personal de apoyo técnico.

Uso de las TIC para la formación judicial y ciudadana, con los proyectos para la gestión del talento humano para el uso de las TIC en el ejercicio judicial ${ }^{10}$ y de Infraestructura TIC para formación en la EJ RLB ${ }^{11}$ y acciones en Gestión del conocimiento con la incorporación de las TIC. (Consejo Superior de la J udicatura, 2012, pp. 9-15)

Como se aprecia, el último eje ratifica la existencia de un particular perfil profesional, el del uso de las TIC en el ejercicio judicial como una necesidad específica de la gestión judicial.

La necesidad de adaptar la gestión judicial a las TIC no proviene solamente de la maduración de la planeación de la rama, sino que también es un imperativo legal: el Decreto 2573 (2014) establece que "La implementación de la estrategia de Gobierno en línea en las ramas legislativa y judicial, en los órganos de control, en los autónomos e independientes y demás organismos del Estado, se realizará bajo un esquema de coordinación y colaboración armónica en aplicación de los principios señalados en artículo 209 de la Constitución Política" (artículo 2, parágrafo) ${ }^{12}$

10 Sobre las posibilidades de la educación jurídica en modalidades a distancia y virtuales (MaDVi) es pertinente la exposición de Parra y Serrano (2019).

11 Sigla para Escuela Judicial Rodrigo Lara Bonilla.

12 Norma incorporada a su vez en el Decreto 1078 de 2015, artículo 2.2.9.1.1.2. 
El primer intento sistemático de hacer efectivas las TIC en la justicia es el Plan Sectorial 2015-2018 (Consejo Superior de la Judicatura, 2015). En él, la política tecnológica ocupa el primer lugar y se compone de cuatro estrategias:

a. Desarrollar el plan estratégico tecnológico de la rama judicial.

b. Rediseñar el sistema de información estadística.

c. Promover e implementar mecanismos para el acceso a la información pública.

Protección de datos judiciales e Implementar el Registro Electrónico de Abogados, Litigantes y Auxiliares de la Justicia (pp. 191-197)

Una vez más encontramos la necesidad de integrar TIC y jurisdicción cuando se verifican los objetivos de la política tecnológica del plan sectorial:

a. Dotar a la rama judicial con recursos tecnológicos para que responda en condiciones de eficiencia a los desafíos que derivan de su razón misional.

b. Apoyar el fortalecimiento del diálogo con la sociedad y la difusión de información a través de las herramientas tecnológicas.

c. Contribuir mediante el uso intensivo de las Tecnologías de la Información y la Comunicación al establecimiento de sistemas avanzados y eficaces de capacitación judicial y formación ciudadana.

Gestionar el cambio tecnológico, con el fin de obtener un mejor rendimiento en la gestión de administración de justicia (p. 196).

La situación planteada es ante todo axiológica, y sus riesgos quedan ilustrados con los recientes casos de detenciones de funcionarios judiciales que manipularon sistemas de reparto de expedientes: en la demanda de Alejandro González Beltrán contra Corficolombiana S. A. (El Tiempo, 2014) el reparto fue manipulado para que el expediente llegara al Juzgado 26 civil del circuito (sistemas Sarj y Bitácora, aparentemente inexpug- 
nables); este mismo año "en el Centro de Servicios de Ejecución de Penas y Medidas de Seguridad de Bogotá, la Fiscalía General de la Nación detectó una supuesta manipulación al sistema de reparto" en el caso de la concesión de una medida de detención domiciliaria a un peligroso narcotraficante, en el que "el ingeniero de la rama judicial, supuestamente, alteró el sistema de reparto de los jueces de ejecución de penas de Bogotá, para que la solicitud del sentenciado quedara en un despacho específico" del procesado (Wradio, 2018). Mucho más reciente y publicitado es el caso de la alteración del reparto dentro del conflicto judicial entre la productora automotriz Hyundai y su distribuidor en Colombia, en la que de nuevo hubo manipulación para que el caso fuera asignado al Juzgado Sexto Civil del Circuito (Coronell, 2018).

\section{Conclusiones}

El destacado desempeño de Colombia en el índice de e-participación implica que el volumen de información que la administración pública pone a disposición del público ya es alto y tiende a crecer.

El destacado desempeño de Colombia en el subíndice de servicios en línea implica que el mantenimiento de ese indicador requerirá un esfuerzo sostenido del Gobierno, máxime si, coherentemente con otras estrategias gubernamentales, se incrementa la participación ciudadana y la demanda por más y mejores servicios gubernamentales en línea, recientemente potenciada por la emergencia COVID.

La permanencia en el tiempo de las iniciativas digitales del Gobierno colombiano, al menos desde 2000 y hasta hoy, indican que es muy probable que los esfuerzos a los que se refieren los puntos anteriores se realicen efectivamente.

La percepción de las TIC en la justicia colombiana ha evolucionado desde la visión meramente archivística hacia una concepción integral de la gestión judicial.

La implementación de las TIC en la justicia ha generado la necesidad de permanente capacitación del personal que trabaja en la rama, pero ese proceso de capacitación aún no ha terminado 
(vista la presencia del mismo en todos los planes) y mantiene su potencial en la medida en que el proceso de asunción tecnológica tampoco se ha consolidado.

Existe todo un dispositivo de planeación social y desarrollo jurídico alrededor de la adaptación de las gestiones administrativa, judicial y privada a las TIC.

El proceso de la e-administración tiene un recorrido y resultados más amplios que los de la e-justicia.

La mayoría de las metas administrativas y judiciales que se ha impuesto el Estado colombiano aún no han sido cumplidas.

\section{Bibliografía}

Almarabeh, T., \& AbuAli, A. (2010). A General Framework for E-Government: Definition Maturity Challenges, Opportunities, and Success. European Journal of Scientific Research, 39(1), 2-42.

Bueno de Mata, F. (2010). E-justicia: Hacia una nueva forma de entender la justicia. Revista Internacional de Estudios de Derecho Procesal y Arbitraje, 1(1), 1-10.

Colombia. Congreso de la República. (2009). Ley 1349, Por la cual se definen principios y conceptos sobre la sociedad de la información y la organización de las Tecnologías de la Información y las Comunicaciones - TIC-, se crea la Agencia Nacional de Espectro y se dictan otras disposiciones. http://www.secretariasenado.gov.co/senado/basedoc/ley_1341_2009.html.

Colombia. Congreso de la República. (2012a). Lè 1564, Por medio de la cual se expide el Código General del Proceso y se dictan otras disposiciones. http://www. secretariasenado.gov.co/senado/basedoc/ley_1564_2012.html.

Colombia. Congreso de la República. (2012b). Ley 1581, Por la cual se dictan disposiciones generales para la protección de datos personales. http://www.secretariasenado.gov.co/senado/basedoc/ley_1581_2012.html.

Colombia. Presidencia de la República. (2014). Decreto 2573, Por el cual se establecen los lineamientos generales de la Estrategia de Gobierno en línea, se reglamenta parcialmente la Ley 1341 de 2009 y se dictan otras disposiciones. https:// www. mintic.gov.co/portal/604/articles-14673_documento.pdf.

Colombia. Presidencia de la República. (2015). Decreto 1078, Por medio del cual se expide el Decreto Único Reglamentario del Sector de Tecnologías de la Información y las Comunicaciones. http://wp.presidencia.gov.co/sitios/normativa/ decretos/2015/decretos2015/decreto\% 201078\% 20del\% 2026\% 20de\% 20 mayo\% 20de\% 202015. pdf.

Colombia. Presidencia de la República. (2020a). Decreto Legislativo 491, Por el cual se adoptan medidas de urgencia para garantizar la atención y la prestación de los servicios por parte de las autoridades públicas y los particulares que cumplan funciones públicas y se toman medidas para la protección laboral y de los contratistas de prestación de servicios de las entidades públicas, en el marco del Estado de Emergencia Económica, Social y Ecológica. https://dapre.presidencia.gov.co/normativa/normativa/Decreto-491-28-marzo-2020.pdf

Colombia. Presidencia de la República. (2020b). Decreto Legislativo 806, Por el cual se adoptan medidas para implementar las tecnologías de la información y las comunicaciones en las actuaciones judiciales, agilizar los procesos judiciales 
y flexibilizar la atención a los usuarios del servicio de justicia, en el marco del Estado de Emergencia Económica, Social y Ecológica. https://dapre.presidencia.gov.co/normativa/normativa/decreto $\% 20806 \% 20$ del $\% 204 \% 20$ de $\% 20$ junio\% 20de\% 202020.pdf

Consejo Nacional de Política Económica y Social. (2000). Documento Conpes 3072. Agenda de Conectividad. Departamento Nacional de Planeación. https://colaboracion.dnp.gov.co/CDT/Conpes/Econ\% C3\%B3micos/3072.pdf

Consejo Superior de la Judicatura. (2002). Plan Sectorial de Desarrollo para la Rama Judicial 2003-2006. Consejo Superior de la Judicatura.

Consejo Superior de la Judicatura. (2006). Plan Sectorial de Desarrollo de la Rama Judicial 2007-2010. Una justicia más eficiente, efectiva y eficaz. Consejo Superior de la Judicatura. http://www.cej.org.co/index.php/component/ docman/doc_download/282-plan-sectorial-de-desarrollo-para-la-rama-judicial-2003-2006? Itemid $=291$

Consejo Superior de la Judicatura. (2011). Plan Sectorial de Desarrollo de la Rama Judicial 2011-2014. Hacia una justicia eficiente, un propósito nacional. Consejo Superior de la Judicatura. https://www.ramajudicial.gov. co/documents/10228/76250/Plan+Sectorial+2011+-+2014. pdf/9c2ad6f7-9ce8-45f8-a281-0eb2751f1e3d

Consejo Superior de la Judicatura. (2012). Plan Estratégico Tecnológico. Rama Judicial de Colombia (Acuerdo PSAA12-9269). Consejo Superior de la Judicatura. http://www.cej.org.co/observatoriocpayca/files/Actos\%20Administrativos/ otros/PSAA12-9269\% 20-\% 20Plan\%20Tecnologico\% 20Anexo.pdf

Consejo Superior de la Judicatura. (2015). Plan Sectorial de Desarrollo Rama Judicial 2015-2018. Consejo Superior de la Judicatura. https://www.ramajudicial.gov. co/documents/1513685/5113559/Plan_Sectorial_de_Desarrollo_Rama_Judicial_2015-2018+(3).pdf/a7b785e1-fb02-4ff6-905 $\bar{b}-\mathrm{c} 16 \mathrm{bac} 93 \mathrm{df} 31 \overline{\overline{2}}$

Coronell, D. (2018, mayo 19). El reparto. https://www.semana.com/opinion/articulo/ carlos-mattos-favorecido-por-corrupcion-en-pleito-con-hyundai-por-daniel-coronell/567808

El Tiempo. (2014, abril 2). Alerta por manipulación de sistemas de Rama Judicial. El Tiempo. http://www.eltiempo.com/archivo/documento/CMS-13476422

Ministerio de Comunicaciones. (2008). Plan Nacional de Tecnologías de la Información y las Comunicaciones 2008-2019. http://eduteka.icesi.edu.co/pdfdir/ColombiaPlanNacionalTIC.pdf

Ministerio de Tecnologías de la Información y las Comunicaciones. (2017). ProyectosEstrategia GEL. Estrategia Gobierno en Línea. http://estrategia.gobiernoenlinea.gov.co/623/w3-propertyvalue-14676.html

Parra Ávila, E., \& Serrano Rubiano, A. L. (2019). Educación jurídica en Colombia en modalidades a Distancia y Virtual: Revisión normativa. En La formación de juristas en la era digital (pp. 30-55). Universidad Nacional Abierta y a Distancia - UNAD. http://hemeroteca.unad.edu.co/index. php/book/article/ view/3514/3435

UN Department of Economic and Social Affairs (UNDESA). (2016). 2016 E-Government for Sustainable Development. United Nations. http://workspace.unpan.org/ sites/Internet/Documents/UNPAN97453.pdf

UN Department of Economic and Social Affairs (UNDESA), \& Civic Resource Group (CRG). (2003). 2003 World Public Sector Report: E-Government at the Crossroads. United Nations. https://publicadministration.un.org/egovkb/portals/ egovkb/Documents/un/2003-Survey/unpan016066.pdf

Wradio. (2018, marzo 1). Efectúan capturas por manipulación de reparto judicial para favorecer a narcotraficante. http://www. wradio.com.co/noticias/judicial/efectuan-capturas-por-manipulacion-de-reparto-judicial-para-favorecer-a-narcotraficante/20180301/nota/3717755.aspx 
